\title{
The Ruled Surfaces According to Bishop Frame in Minkowski 3-Space
}

\author{
Nural Yüksel \\ Department of Mathematics, Erciyes University, 38039 Kayseri, Turkey \\ Correspondence should be addressed to Nural Yüksel; yukseln@erciyes.edu.tr
}

Received 8 April 2013; Accepted 18 June 2013

Academic Editor: Chuanzhi Bai

Copyright (C) 2013 Nural Yüksel. This is an open access article distributed under the Creative Commons Attribution License, which permits unrestricted use, distribution, and reproduction in any medium, provided the original work is properly cited.

We investigate the ruled surfaces generated by a straight line in Bishop frame moving along a spacelike curve in Minkowski 3-space. We obtain the distribution parameters, mean curvatures. We give some results and theorems related to be developable and minimal of them. Furthermore, we show that, if the base curve of the ruled surface is also an asymtotic curve and striction line, then the ruled surface is developable.

\section{Introduction}

Recently, the theory of surfaces and their transformations has been studied extensively in differential geometry. The ruled surfaces have been a powerful subject in the Minkowski space $\mathbb{R}_{1}^{3}$ for line geometry for a long time. In the literature, Kobayashi [1] was the first author to address this problem and examined minimal spacelike ruled surfaces in the Minkowski $\mathbb{R}_{1}^{3}$. Kim and Yoon [2] have classified the Lorentz surfaces.

Izumiya and Takeuchi [3] obtained some characterizations for ruled surfaces. Turgut and Hacisalihoğlu [4, 5] defined spacelike ruled surfaces and obtained some characterizations in the three-dimensional Minkowski space. Yaylı [6] obtained the distribution parameter of a spacelike ruled surface generated by a spacelike straight line in Frenet frame along a spacelike curve. Yaylı and Saracoglu $[7,8]$ studied timelike and spacelike developable ruled surfaces in Minkowski space. Orbay and Aydemir [9] obtained the distrubition parameter, mean curvature, and Gaussian curvature, and some new results and theorems were given for developable and minimal spacelike ruled surfaces.

In this paper, making use of the method in a paper of Yaylı [6], we obtained some characterizations for spacelike Ruled surfaces according to Bishop frame in Minkowski 3-space.

\section{Preliminaries}

Let $\mathbb{R}_{1}^{3}$ be a Minkowski 3-space with the metric tensor $I=$ $\langle\cdot, \cdot\rangle=d x_{1}^{2}-d x_{2}^{2}+d x_{3}^{2}$. The norm of $v \in \mathbb{R}_{1}^{3}$ is defined by $\|v\|=\sqrt{|\langle v, v\rangle|}$. A vector $v \in \mathbb{R}_{1}^{3}$ is said to be spacelike if $\langle v, v\rangle>0$ or $v=0$, timelike if $\langle v, v\rangle<0$, and lightlike (or null) if $\langle v, v\rangle=0$ and $v \neq 0$.

Let $\alpha: I \rightarrow \mathbb{R}_{1}^{3}, \alpha(s)=\left(\alpha_{1}(s), \alpha_{2}(s), \alpha_{3}(s)\right)$ be a smooth regular curve in $\mathbb{R}_{1}^{3}$. We say that $\alpha$ is a spacelike (resp. timelike, lightlike) if $\alpha^{\prime}(t)$, a spacelike (resp. timelike, lightlike) vector for all $s \in I \subset \mathbb{R}$.

A surface in the Minkowski 3-space is called a spacelike surface if the Lorentz metric on the surface is a positive definite [10]. A ruled surface is a surface swept out by a straight line $X$ moving along a curve $\alpha$. The various positions of the generating line $X$ are called the rullings of the surface. Such a surface has a parametrization in the ruled form as follows:

$$
\phi(s, v)=\alpha(s)+v X(s),
$$

where $\alpha$ is the base curve and $X$ is the director vector along $\alpha$. If the tangent plane is constant along a fixed rulling, then the ruled surface is called a developable surface. The remaining ruled surfaces are called skew surfaces [4]. The spacelike ruled surface $M$ in $\mathbb{R}_{1}^{3}$ is given by the parametrization

$$
\begin{aligned}
\phi: I \times \mathbb{R} & \longrightarrow \mathbb{R}_{1}^{3} \\
(s, v) & \longrightarrow \phi(s, v)=\alpha(s)+v X(s),
\end{aligned}
$$

where $\alpha: I \rightarrow \mathbb{R}_{1}^{3}$ is a differentiable spacelike curve parametrized by its arc length in $\mathbb{R}_{1}^{3}$ and $X(s)$ is the director vector 
of the director curve such that $X$ is ortogonal the tangent vector field $T$ of the base curve $\alpha$.

Denote by $\{T, N, B\}$ the moving Frenet frame along the regular curve with arc-lenght parameter $s$. The Frenet trihedron consists of the tangent vector $T$, the principal normal vector $N$, and the binormal vector $B$. If $\alpha$ is a spacelike curve with a spacelike binormal, then the Frenet frame has the following properties:

$$
\begin{aligned}
& T^{\prime}(s)=\varkappa(s) N, \\
& N^{\prime}(s)=\varkappa(s) T(s)+\tau(s) B(s), \\
& B^{\prime}(s)=\tau(s) N(s),
\end{aligned}
$$

where

$$
\langle T, T\rangle=1, \quad\langle N, N\rangle=-1, \quad\langle B, B\rangle=1 .
$$

The Bishop frame or parallel transport frame is an alternative approach to defining a moving frame that is well defined even when the curve has a vanishing second derivative. One can express parallel transport of an orthonormal frame along a curve simply by parallel transporting each component of the frame. The tangent vector and any convenient arbitrary basis for the remainder of the frame are used.

Let us consider the Bishop frame $\left\{T, N_{1}, N_{2}\right\}$ of the spacelike curve $\alpha(s)$ such that $T(s)$ the spacelike unit tangent vector, $N_{1}(s)$ is timelike unit normal vector, and $N_{2}(s)$ the spacelike unit binormal vector. So scalar product and cross product of the vectors $\left\{T, N_{1}, N_{2}\right\}$ are given by

$$
\begin{aligned}
& \langle T, T\rangle=-\left\langle N_{1}, N_{1}\right\rangle=\left\langle N_{2}, N_{2}\right\rangle=1, \\
& \left\langle T, N_{1}\right\rangle=\left\langle T, N_{2}\right\rangle=\left\langle N_{1}, N_{2}\right\rangle=0, \\
& T \wedge N_{1}=N_{2}, \\
& N_{1} \wedge N_{2}=T, \\
& N_{2} \wedge T=-N_{1} .
\end{aligned}
$$

The Bishop frame $\left\{T, N_{1}, N_{2}\right\}$ is expressed as

$$
\begin{aligned}
& T^{\prime}(s)=k_{1} N_{1}(s)-k_{2} N_{2}(s), \\
& N_{1}^{\prime}(s)=k_{1} T(s), \\
& N_{2}^{\prime}(s)=k_{2} T(s) .
\end{aligned}
$$

One can show that

$$
\begin{gathered}
\kappa=\sqrt{\left|k_{2}^{2}-k_{1}^{2}\right|}, \\
\tau=\frac{d \theta}{d s}, \quad \theta(s)=\operatorname{argtanh} \frac{k_{2}}{k_{1}}
\end{gathered}
$$

so that $k_{1}$ and $k_{2}$ effectively correspond to a cartesian coordinate system for the polar coordinates $\kappa, \theta$ with $\theta=$ $\int \tau(s) d s[11]$.

Remark 1. From the definition of the argtanh function we assume that $\left|k_{2} / k_{1}\right|<1$.
The distribution parameter, the mean curvature, and the Gaussian curvature of the ruled surface $\phi(s, v)$ are given by

$$
\begin{gathered}
P_{x}=\frac{\operatorname{det}\left(T, X, D_{T} X\right)}{\left\langle D_{T} X, D_{T} X\right\rangle}, \\
H=\frac{1}{2}\left[\frac{G l+E n-2 F m}{E G-F^{2}}\right],
\end{gathered}
$$

where $D$ is the Levi-Civita connection on $\mathbb{R}_{1}^{3}$.

Theorem 2. A spacelike ruled surface is a developable surface if and only if the distrubition parameter of the spacelike ruled surface is zero [4].

The foot on the main rulling of the common perpendicular of two constructive rullings in the ruled surface is called a central point. The locus of the central point is called the striction curve. The parametrization of the striction curve on the ruled surface is given by

$$
\bar{\alpha}(s)=\alpha(s)-\frac{\left\langle T, D_{T} X\right\rangle}{\left\langle D_{T} X, D_{T} X\right\rangle} X(s) .
$$

\section{One Parameter Spatial Motion in $\mathbb{R}_{1}^{3}$}

Let $\alpha: I \rightarrow \mathbb{R}_{1}^{3}$ be a spacelike curve and $\left\{T, N_{1}, N_{2}\right\}$ be its Bishop frame where $T, N_{1}$, and $N_{2}$ are the tangent, principal normal, and binormal vectors of the curve $\alpha$, respectively. $T$ and $N_{2}$ are spacelike vectors and $N_{1}$ is a timelike vector.

The two coordinate systems $\left\{O ; T, N_{1}, N_{2}\right\}$ and $\left\{O^{\prime} ; e_{1}\right.$, $\left.e_{2}, e_{3}\right\}$ are orthogonal coordinate systems in $\mathbb{R}_{1}^{3}$ which represent the moving space $H$ and the fixed space $H^{\prime}$, respectively.

Let $X$ be a unit spacelike vector

$$
\begin{aligned}
& X \in \operatorname{Sp}\left\{T(s), N_{1}(s), N_{2}(s)\right\}, \\
& X=x_{1} T(s)+x_{2} N_{1}(s)+x_{3} N_{2}(s),
\end{aligned}
$$

such that

$$
\langle X, X\rangle=1
$$

We can obtain the distrubition parameter of the spacelike ruled surface generated by a straight line $X$ of the moving space $H$. Differentiating (13) with respect to $s$, we get

$$
\begin{array}{r}
D_{T} X=x_{1} T^{\prime}(s)+x_{2} N_{1}^{\prime}(s)+x_{3} N_{2}^{\prime}(s), \\
x_{1}^{2}-x_{2}^{2}+x_{3}^{2}=1 .
\end{array}
$$

By using the Bishop frame in (15), we obtain

$$
D_{T} X=\left(x_{2} k_{1}+x_{3} k_{2}\right) T(s)+x_{1} k_{1} N_{1}(s)-x_{1} k_{2} N_{2}(s) .
$$

From (10) we get

$$
\begin{array}{r}
P_{x}=-\frac{x_{1}\left(x_{2} k_{2}+x_{3} k_{1}\right)}{\left(x_{2} k_{1}+x_{3} k_{2}\right)^{2}+x_{1}^{2}\left(k_{2}^{2}-k_{1}^{2}\right)}, \\
x_{1}^{2}-x_{2}^{2}+x_{3}^{2}=1 .
\end{array}
$$


Theorem 3. Let $M$ be a spacelike ruled surface given by the parametrization (2). $M$ is developable if and only if either the director vector $X$ lies in the plane generated by $N_{1}(s)$ and $N_{2}(s)$ or the base curve $\alpha$ is a planar curve such that the curvatures of $\alpha, k_{1}$ and $k_{2}$ satisfy

$$
\frac{k_{1}}{k_{2}}=-\frac{x_{2}}{x_{3}}
$$

Proof. Let $M$ be a ruled surface. By using (17) and Theorem 2,

$$
x_{1}\left(x_{2} k_{2}+x_{3} k_{1}\right)=0
$$

is obtained. In that case, we have

$$
x_{1}=0 \text { or }\left(x_{2} k_{2}+x_{3} k_{1}\right)=0 .
$$

Thus

$$
\begin{aligned}
& X(s) \in \operatorname{Sp}\left\{N_{1}(s), N_{2}(s)\right\} \\
& \text { or } \frac{k_{1}}{k_{2}}=-\frac{x_{2}}{x_{3}}=\text { constant. }
\end{aligned}
$$

From (9), we get

$$
\tau=0 .
$$

So, $\alpha$ is a planar curve. This completes the proof.

\section{Special Cases}

Let $M$ be a spacelike ruled surface given by the parametrization (2), and, $X$ be the director vector of the base curve $\alpha$.

4.1. The Case $X=T$ (Spacelike). In this case, $x_{1}=1, x_{2}=$ $x_{3}=0$ thus from (17)

$$
P_{T}=0 \text {. }
$$

Hence the following theorem is hold.

Theorem 4. During the one-parameter spatial motion $H / H^{\prime}$ the spacelike ruled surface in the fixed space $H^{\prime}$ generated by the tangent line $T$ of the curve $\alpha(s)$ in the moving space $H$ is developable.

4.2. The Case $X=N_{2}$ (Spacelike). From Theorem 3, it is obvious that $P_{N_{2}}=0$.

4.3. The Case $X \in \operatorname{Sp}\left\{T(s), N_{1}(s)\right\}$. In this case, $x_{3}$ is zero. So, the director vector $X$ is given by

$$
X=x_{1} T+x_{2} N_{1}, \quad x_{1}^{2}-x_{2}^{2}=1 .
$$

The distribution parameter of the ruled surface is given by

$$
\begin{aligned}
P_{x} & =-\frac{x_{1} x_{2} k_{2}}{x_{2}^{2} k_{1}^{2}+x_{1}^{2}\left(k_{2}^{2}-k_{1}^{2}\right)} \\
& = \pm \frac{x_{2} \sqrt{1+x_{2}^{2}} k_{2}}{x_{2}^{2} k_{1}^{2}+\left(1+x_{2}^{2}\right)\left(k_{2}^{2}-k_{1}^{2}\right)} .
\end{aligned}
$$

The ruled surface is developable if and only if $P_{x}=0$. Thus

$$
x_{2}=0 \text { or } k_{2}=0 \text {. }
$$

If $x_{2}=0$, this is case 4.1. If the second curvature $k_{2}$ is zero, then we can say that the base curve $\alpha$ is a planar curve.

4.4. The Case $X \in \operatorname{Sp}\left\{T(s), N_{2}(s)\right\}$. In this case, $x_{2}$ is zero. So, the director vector $X$ is given by

$$
X=x_{1} T+x_{3} N_{2}, \quad x_{1}^{2}+x_{3}^{2}=1 .
$$

From (17) the distribution parameter is obtained as

$$
P_{x}=-\frac{x_{1} x_{3} k_{1}}{k_{2}^{2}-x_{1}^{2} k_{1}^{2}}
$$

$P_{x}=0$ if and only if

$$
x_{1}=0 \quad \text { or } \quad x_{3}=0 .
$$

If $x_{1}$ is zero, this is case 4.3. If $x_{3}$ is zero, this is The case 4.1.

From Theorem 3, $M$ is a developable spacelike ruled surface.

4.5. The Case $X \in \operatorname{Sp}\left\{N_{1}(s), N_{2}(s)\right\}$. From Theorem 3 it is obvious that the spacelike ruled surface is developable.

By using (11) we compute the mean curvatures of the spacelike ruled surfaces generated by spacelike vectors $T(s), N_{2}(s)$, and $X$.

Proposition 5. Let $M_{T}$ be a spacelike ruled surface generated by the tangent line $T$ of the curve $\alpha$. From (11) the mean curvature is obtained as follows:

$$
H_{T}=\frac{1}{2} \frac{\varepsilon\left(k_{1} k_{2}^{\prime}-k_{2} k_{1}^{\prime}\right)}{v \sqrt{\left|k_{1}^{2}-k_{2}^{2}\right|}}, \quad \epsilon= \pm 1
$$

Thus from (6) we have

$$
H_{T}=-\frac{1}{2} \frac{\varepsilon}{v} \frac{\tau}{\kappa}
$$

Corollary 6. The surface $M_{T}$ is minimal if and only if $\alpha$ is a planar curve.

Proof. Let $M_{T}$ be minimal. In this case, from (31), we get

$$
\tau=0
$$

Conversely, let $\alpha$ be a planar curve. Then $\tau=0$ implies that $H_{T}=0$. This completes the proof.

Proposition 7. Let $M_{N_{2}}$ be a spacelike ruled surface generated by the binormal line $\mathrm{N}_{2}$ of the base curve $\alpha$. From (11) the mean curvature is obtained as follows:

$$
H_{N_{2}}=-\frac{\varepsilon k_{1}}{2\left(1+v k_{2}\right)}, \quad \varepsilon= \pm 1 .
$$

So, the following result may be given. 
Corollary 8. According to the Bishop frame, there is no minimal spacelike ruled surface generated by the binormal line $N_{2}$ in $\mathbb{R}_{1}^{3}$.

Proposition 9. Let $M$ be a spacelike ruled surface which is given by the parametrization (2):

$$
H_{x}=\frac{1}{2}\left[\frac{G l+E n-2 F m}{E G-F^{2}}\right] \text {, }
$$

where

$$
\begin{aligned}
& E=\left\langle\phi_{s}, \phi_{s}\right\rangle=\left(1+v\left(k_{1} x_{2}+k_{2} x_{3}\right)\right)^{2}+v^{2} k_{1}^{2}\left(k_{2}^{2}-k_{1}^{2}\right), \\
& F=\left\langle\phi_{s}, \phi_{v}\right\rangle=x_{1} \text {, } \\
& G=\left\langle\phi_{v}, \phi_{v}\right\rangle=1 \text {, } \\
& l=\frac{1}{\left\|\phi_{s} \times \phi_{v}\right\|}\left\langle\phi_{s s}, \phi_{s} \times \phi_{v}\right\rangle \\
& =\frac{1}{\left\|\phi_{s} \times \phi_{v}\right\|} \\
& \times\left[\begin{array}{c}
-\left(1+v\left(x_{2} k_{1}+x_{3} k_{2}\right)\right)^{2}\left(x_{3} k_{1}+x_{2} k_{2}\right) \\
-\left(1+v\left(x_{2} k_{1}+x_{3} k_{2}\right)\right) v x_{1}\left(x_{3} k_{1}^{\prime}+x_{2} k_{2}^{\prime}\right) \\
+v^{2} x_{1}^{3}\left(k_{1} k_{2}^{\prime}-k_{1}^{\prime} k_{2}\right) \\
+v^{2} x_{1}\left(x_{2} k_{1}+x_{3} k_{2}\right)\left(x_{2} k_{1}^{\prime}+x_{3} k_{2}^{\prime}\right) \\
+v^{2} x_{1}^{2}\left(x_{3} k_{1}+x_{2} k_{2}\right)\left(k_{1}^{2}-k_{2}^{2}\right)
\end{array}\right] \\
& m=\frac{1}{\left\|\phi_{s} \times \phi_{v}\right\|}\left\langle\phi_{s v}, \phi_{s} \times \phi_{v}\right\rangle=\frac{-x_{1}\left(k_{1} x_{3}+k_{2} x_{2}\right)}{\left\|\phi_{s} \times \phi_{v}\right\|}, \\
& n=\frac{1}{\left\|\phi_{s} \times \phi_{v}\right\|}\left\langle\phi_{v v}, \phi_{s} \times \phi_{v}\right\rangle=0,
\end{aligned}
$$

where

$$
\begin{aligned}
N & =\frac{\phi_{s} \times \phi_{v}}{\left\|\phi_{s} \times \phi_{v}\right\|} \\
& =\frac{1}{\left\|\phi_{s} \times \phi_{v}\right\|}\left\{\begin{array}{c}
{\left[v x_{1}\left(x_{3} k_{1}+x_{2} k_{2}\right)\right] T} \\
+\left[x_{3}\left(1+v\left(x_{2} k_{1}+x_{3} k_{2}\right)\right)+v x_{1}^{2} k_{2}\right] N_{1} \\
+\left[x_{2}\left(1+v\left(x_{2} k_{1}+x_{3} k_{2}\right)\right)-v x_{1}^{2} k_{1}\right] N_{2}
\end{array}\right\}
\end{aligned}
$$

is a unit normal vector of the spacelike ruled surface $M$.

Proposition 10. Let $M$ be a spacelike ruled surface given by the parametrization (2). If the base curve of $M$ is also a striction curve, then the curvature functions $k_{1}$ and $k_{2}$ of the base curve $\alpha$ satisfy the following equation:

$$
x_{2} k_{1}+x_{3} k_{2}=0
$$

Proof. Let the base curve $\alpha$ be the striction curve. Thus, from (12),

$$
\left\langle T, D_{T} X\right\rangle=0
$$

Then we have

$$
x_{2} k_{1}+x_{3} k_{2}=0 \text {. }
$$

Hence the following result holds.

Corollary 11. Let $M$ be a spacelike ruled surface given by the parametrization (2). If the base curve of $M$ is also striction curve, then $\alpha$ is a planar curve.

Proof. Let the base curve $\alpha$ be also striction curve. Thus from (42)

$$
x_{2} k_{1}+x_{3} k_{2}=0 \text {. }
$$

Hence we get

$$
\frac{k_{1}}{k_{2}}=-\frac{x_{3}}{x_{2}}=\text { constant. }
$$

From (9), $\alpha$ is a planar curve.

Proposition 12. Let $M$ be a spacelike ruled surface given by the parametrization (2). If the base curve of $M$ is also asymtotic curve, then

$$
\left(1+v\left(x_{2} k_{1}+x_{3} k_{2}\right)\right)\left(x_{2} k_{2}+x_{3} k_{1}\right)=0 .
$$

Proof. We assume that the base curve of the surface $M$ is the asymtotic curve. In that case,

$$
\left\langle\alpha^{\prime \prime}, N\right\rangle=0 \text {. }
$$

From (46), we have

$$
\left(1+v\left(x_{2} k_{1}+x_{3} k_{2}\right)\right)\left(x_{2} k_{2}+x_{3} k_{1}\right)=0 .
$$

Theorem 13. Let the base curve of the surface $M$ be an asymtotic curve. If the base curve of $M$ is also a striction curve, the spacelike ruled surface $M$ is developable.

Proof. Let the base curve of the surface $M$ be both an asymtotic curve and striction curve. By using (42) and (45) we obtain

$$
\left(x_{2} k_{2}+x_{3} k_{1}\right)=0 .
$$

From (17), the surface is developable.

Proposition 14. Let $M$ be a spacelike ruled surface given by the parametrization (2). We obtain the following results for the spacelike ruled surfaces.

(i) The s-parameter curve of $M$ is also an asymtotic curve if and only if

$$
\left[\begin{array}{c}
-\left(1+v\left(x_{2} k_{1}+x_{3} k_{2}\right)\right)^{2}\left(x_{3} k_{1}+x_{2} k_{2}\right) \\
-\left(1+v\left(x_{2} k_{1}+x_{3} k_{2}\right)\right) v x_{1}\left(x_{3} k_{1}^{\prime}+x_{2} k_{2}^{\prime}\right) \\
+v^{2} x_{1}^{3}\left(k_{1} k_{2}^{\prime}-k_{1}^{\prime} k_{2}\right) \\
+v^{2} x_{1}\left(x_{2} k_{1}+x_{3} k_{2}\right)\left(x_{2} k_{1}^{\prime}+x_{3} k_{2}^{\prime}\right) \\
+v^{2} x_{1}^{2}\left(x_{3} k_{1}+x_{2} k_{2}\right)\left(k_{1}^{2}-k_{2}^{2}\right)
\end{array}\right]=0 .
$$


(ii) The v-parameter curve of $M$ is also an asymtotic curve.

Proof. (i) If the $s$-parameter curve of $M$ is also an asymtotic curve, then

$$
\left\langle\phi_{s s}, N\right\rangle=0
$$

From (36), we obtain (46).

(ii) If the $v$-parameter curve of $M$ is also an asymtotic curve, then

$$
\left\langle\phi_{v v}, N\right\rangle=0
$$

$v$-parameter curve of $M$ is an asymtotic curve.

Theorem 15. Let $M$ be a developable spacelike ruled surface given by the parametrization (2). The s-parameter curve of $M$ is also asymtotic curve if and only if $M$ is a minimal surface.

Proof. Assume that $s$-parameter curve of the surface $M$ an asymtotic curve. Then

$$
l=\left\langle\phi_{s s}, N\right\rangle=0
$$

where $N$ is a unit normal vector field of the surface $M$. Since $M$ is a developable ruled surface,

$$
x_{1}\left(x_{2} k_{1}+x_{3} k_{2}\right)=0
$$

Thus from (34)

$$
H_{x}=0
$$

is obtained.

Conversely, let $M$ be a minimal surface. From (14), we get

$$
l-2 F m=0
$$

since $M$ is a developable ruled surface, we obtain

$$
l=0 \text {. }
$$

This completes the proof.

\section{References}

[1] O. Kobayashi, "Maximal surfaces in the 3-dimensional Mink-

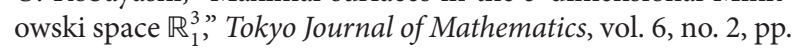
297-309, 1983.

[2] Y. H. Kim and D. W. Yoon, "Classification of ruled surfaces in Minkowski 3-spaces," Journal of Geometry and Physics, vol. 49, no. 1, pp. 89-100, 2004.

[3] S. Izumiya and N. Takeuchi, "Special curves and ruled surfaces," Beiträge zur Algebra und Geometrie, vol. 44, no. 1, pp. 203-212, 2003.

[4] A. Turgut and H. H. Hacısalihoğlu, "On the distribution parameter of timelike ruled surfaces in the Minkowski 3-space," Far East Journal of Mathematical Sciences, vol. 5, no. 2, pp. 321-328, 1997.
[5] A. Turgut and H. H. Hacısalihoğlu, "Spacelike ruled surfaces in Minkowski 3-Space," Communications de la Faculté des Sciences de l'Université d'Ankara Séries A, vol. 1, p. 46, 1997.

[6] Y. Yayl1, "On the motion of the Frenet vectors and spacelike ruled surfaces in the Minkowski 3-space," Mathematical of Computational Applications, vol. 5, no. 1, pp. 49-55, 2000.

[7] Y. Yaylı and S. Saracoglu, "On developable ruled surfaces in Minkowski space," Advances in Applied Clifford Algebras, vol. 22, no. 2, pp. 499-510, 2012.

[8] Y. Yaylı and S. Saracoglu, "On developable ruled surfaces," Algebras, Groups and Geometries, vol. 28, no. 2, pp. 165-183, 2011.

[9] K. Orbay and I. Aydemir, "The ruled surfaces generated by frenet vectors of a curve in $\mathbb{R}_{1}^{3}$," C.B.U. Journal of Science, vol. 6.2, pp. 155-160, 2010.

[10] R. Lopez, "Differential geometry of curves and surfaces in Lorentz-Minkowskispace," http://128.84.158.119/abs/0810.3351v1.

[11] B. Bukcu and M. K. Karacan, "Bishop frame of the spacelike curve with a spacelike binormal in Minkowski 3-space," Selçuk Journal of Applied Mathematics, vol. 11, no. 1, pp. 15-25, 2010. 


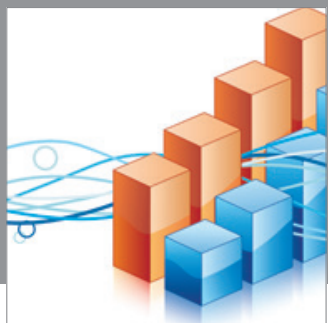

Advances in

Operations Research

mansans

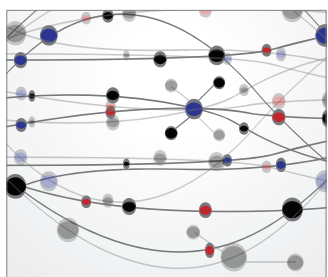

The Scientific World Journal
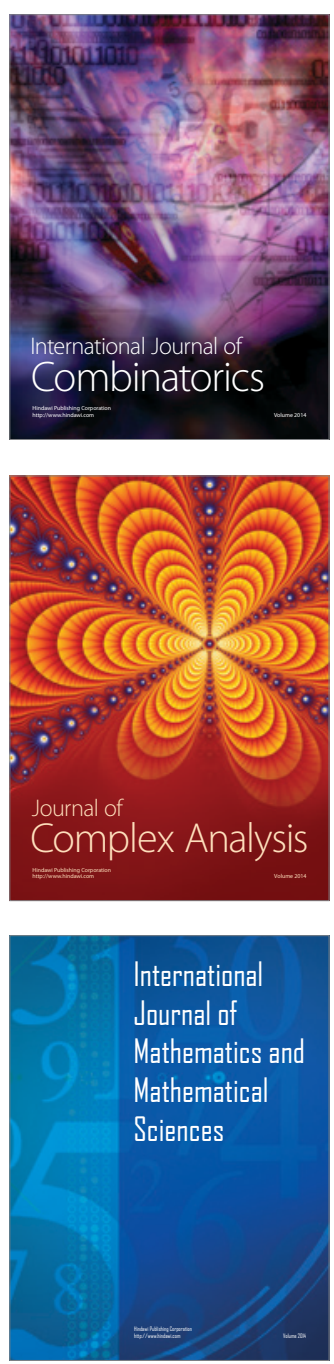
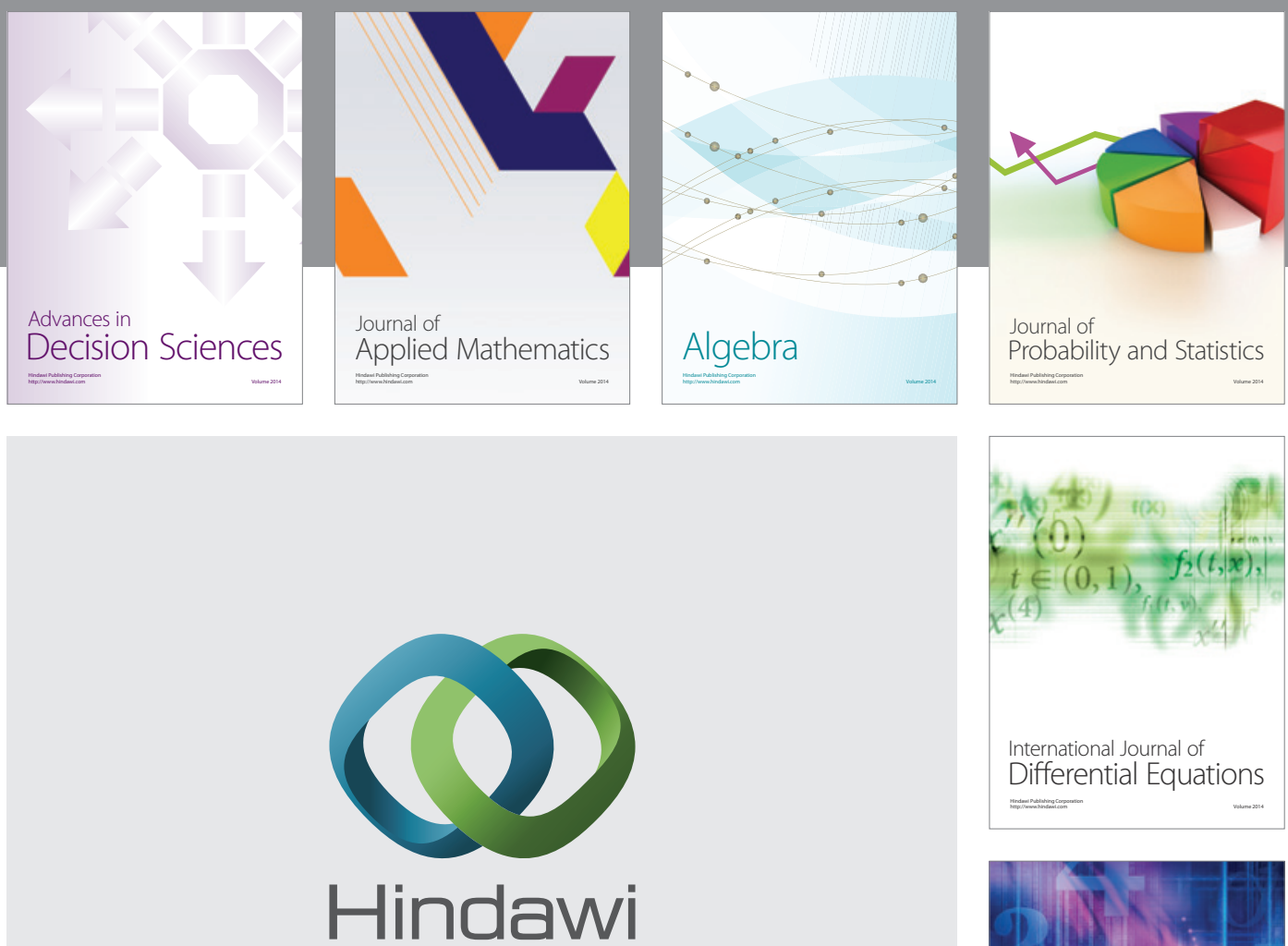

Submit your manuscripts at http://www.hindawi.com
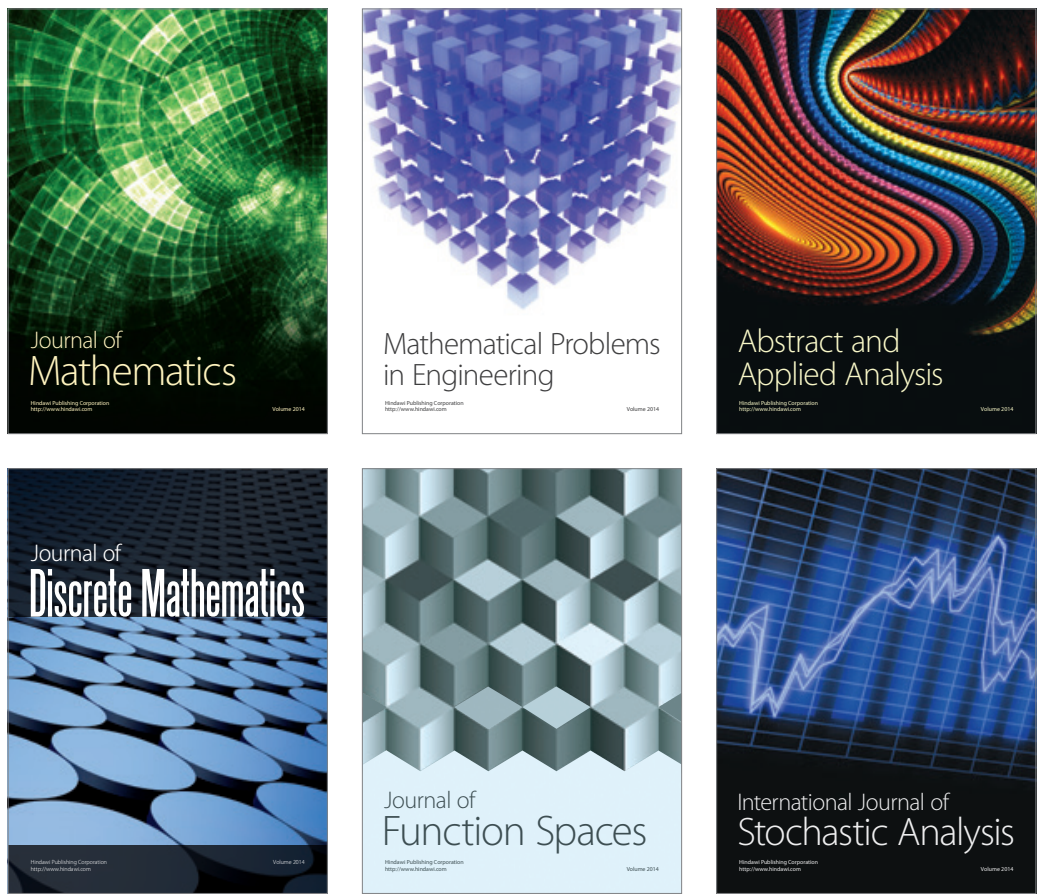

Journal of

Function Spaces

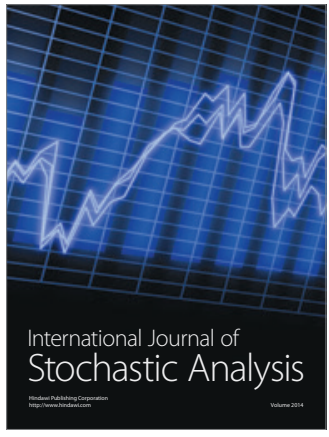

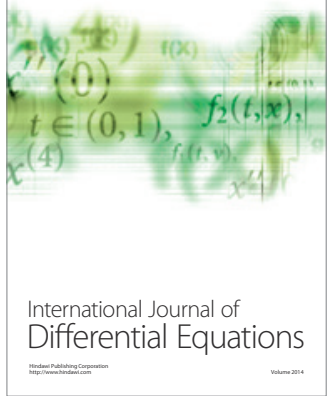
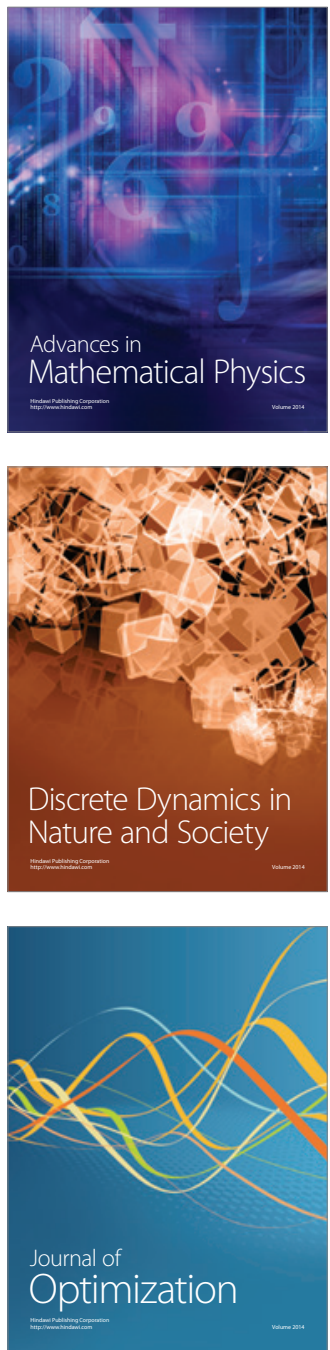